\title{
Study on the Key Problems of UHVAC/DC Power Grids with New Generation Large Synchronous Condenser
}

\author{
Xiao Fan, Zhou Kunpeng, Wang Tao, Cao Kan, Rao Yuze \\ Hubei Electric Power Research Institute, SGCC, Wuhan, China
}

\begin{abstract}
In order to cope with the great pressure caused by the gradually exhaustion of fossil energy and environmental protection and climate warming, the development and application of the renewable energy has become an important foundation and development direction in the field of energy. However, due to the impact of energy and resource endowment, China's load center and renewable energy base into the characteristics of long-range reverse distribution, the use of long-distance large-capacity transmission potential is necessary. With the "strong DC system and weak AC system" problem of the company power grid is increasingly prominent, the higher demand of dynamic reactive power support is put forward in the UHV DC power transmission project. Then, the large-capacity dynamic reactive power of new generation synchronous condenser is large-scale built up in the UHV DC/AC power system.

Due to the high requirement of response speed and capacity in the UHV DC power transmission, the structure, dynamic characteristics, excitation control and relay protection and other aspects of the large synchronous condenser are different from generators and traditional synchronous condenser. Based on this, the dynamic reactive power demand of UHV DC power system is analyzed in this paper. Then, the main situation of large synchronous condenser is considered. In addition, the key points of the excitation control system and protection system are also discussed in this paper. There are important theoretical and practical significance for the safe and reliable operation of the UHV DC/AC power system.
\end{abstract}

\section{Introduction}

In order to cope with the great pressure caused by the gradually exhaustion of fossil energy and environmental protection and climate warming, the development and application of the renewable energy has become an important foundation and development direction in the field of energy. However, due to the impact of energy and resource endowment, China's load center and renewable energy base into the characteristics of longrange reverse distribution, the use of long-distance largecapacity transmission potential is necessary. However, the conventional DC converter station needs to consume a large amount of reactive power, which is mainly provided by the static reactive power compensation device in the converter station. The reactive power absorbed by the DC converter station can be significantly increased when a large disturbance is occurred in the power system. While the dynamic reactive power compensation capacity of the power grid will be lacked and the risk of voltage instability is greatly increased due to the output of the static reactive power compensation device is reduced. Compared with the static reactive power compensation device, the capacity of the synchronous power converter compensation is large. In addition, the forced excitation of the excitation system can be implemented automatically under grid voltage is drop condition. Therefore, with the "strong DC system and weak AC system" problem of the company power grid is increasingly prominent, it is of great significance to improve the stability of grid voltage and dynamic reactive power support and very important for the safe and stable operation of UHV AC-DC hybrid power system with the construction process of large synchronous condensers in UHV power system is accelerated.

According to the national grid company planning, there are 66 new generation large synchronous condenser will be deployed in the power system. However, due to the high requirement of response speed and capacity in the UHV DC power transmission, the structure, dynamic characteristics, excitation control and relay protection and the aspects of the large synchronous condenser are different from generators and traditional synchronous condenser.

For the voltage stability, the influencing factors, analytical methods, stabilization criteria and coping measures, the voltage stability problem of DC feed power grid, especially multi-DC feed power grid, are systematically expounded in [1]-[4]. In order to improve the static voltage stability of the receiving power grid and to prevent the transient voltage instability, a load optimization control method is proposed in[5-7].For multiple DC are feed in Shanghai power grid and east China power grid and the southern power grid, the impact of large disturbance are analyzed and the 
measures, such as, static var compensator (SVC), static synchronous compensator (STATCOM) and synchronous condenser are proposed to improve the transient voltage stability of the power system in the literature [8-11].

For the excitation control system, the excitation system has a significant effect on the fast response performance and reactive power output characteristics of large-scale synchronous condenser. At present, the study of excitation control mainly focuses on synchronous generators. Meanwhile, there is no large-scale synchronous camera excitation control system related to the application practice. In addition, although the main part of of the excitation control system in the large-scale synchronous condenser should be the same with the same capacity generator, but its use of "idling" grid-way, as well as in the low excitation and excitation conditions under the special needs, making new changes have taken place in the excitation control system of the large-scale synchronous condenser.

For the relay protection system, the relay protection configuration scheme of the the large-scale synchronous condenser under the static frequency conversion (SFC) startup mode is proposed. This paper mainly introduces the current and voltage analog quantity input to the relay protection device, which is the obvious low frequency characteristic, and its frequency is changing from $0 \sim 50 \mathrm{~Hz}$. It is pointed out that the low-frequency characteristics should be considered in the protection configuration of the large-scale synchronous condenser. However, a new generation of large condenser new changes not only have taken place in the startup mode, at the same time in the condenser standardization of relay protection design, configuration and setting technical standards lack of corresponding standards. In addition, there are may be a new problem in the excitation-loss protection of the large-scale synchronous condenser. Based on this, the dynamic reactive power demand of UHV DC power system is analyzed in this paper. Then, the main situation of large synchronous condenser is considered. In addition, the key points of the excitation control system and protection system are also discussed in this paper. There are important theoretical and practical significance for the safe and reliable operation of the UHV DC/AC power system.

\section{Voltage stability of UHV power grid}

\subsection{Voltage stability of DC inverter station}

\subsubsection{The prominent problem of dynamic reactive power shortage in the end of the power grid}

DC system rectifier side using fixed power control, inverter side using the set out arc angle control strategy. Under a voltage drop is occurred in the end of AC system condition, the DC voltage will be reduced. In order to ensure the DC power is constant, the DC current will be increased by the rectifier station. An increase in the DC current will result in a reactive gain increase in the endfed converter. When the voltage of the AC system is reduced, the reactive power of the AC filter will be reduced. The above two points lead to a decrease in the AC voltage after the converter station from the system to absorb the reactive power increases, the stability of voltage is easy to be lose.

\subsubsection{DC feed reduces the system's dynamic reactive power reserve}

Under normal conditions, the reactive power exchange between the UHV DC converter station and the system is zero. When 8 million kilowatts of rated power are supplied by DC power, the total active is distributed to the end of the $220 \mathrm{kV}$ power grid, and 1.2 million reactive power are consumed by $500 \mathrm{kV}$ step-down transformer. Under the same power consumption condition, if 12 sets of 66 million kilowatts generator instead of DC feed in the end of the grid, about 1.9 million kilowatts of reactive power can be provided by the system. In the dynamic process of the system, a large number of dynamic reactive power can be provided to support the system voltage. Compared to conventional generators, DC large-scale feed reduces the system dynamic reactive power reserve.

\subsubsection{DC feed-in deteriorate the voltage system characteristic}

Under the AC system voltage decreases and DC feed into the end of the grid condition, the conventional generating units will increase the reactive power output and reactive power will be absorb by the DC inverter station from the system. According to the AC bus voltage reduced by $1 \%$, DC inverter station will absorb 50,000 kvars of reactive power from the system, the same size of the conventional power supply to the system at least 300,000 kvars of reactive power will be provided by the system. Conventional power supply and dc inverter station in the system voltage and reduce the reactive voltage regulating characteristics, on the other hand, the voltage regulation system characteristic are deteriorated due to the DC feedin.

\subsubsection{Dc feed-in reduces the resistance of the system reactive power}

As the DC large-scale feed to replace the conventional power supply, the short-circuit capacity decreased in the end of the system, resulting in the system anti-reactive impact capacity weakened in the DC multi-feed area is particularly evident. For example, by the end of the power grid, 8 million kw dc into the equivalent of 12 sets of $660000 \mathrm{kw}$ conventional unit, compared with the conventional unit, ac system short circuit current will reduce about 20000 due to the dc feed-in. According to the reactive power impact, about $60 \mathrm{kV}$ voltage fluctuation will increased due to 4 million dc feed in the backend system. It's means that the system voltage is collapsed. 


\subsubsection{DC fault (commutation failure) to absorb a large number of reactive power}

Under single UHV dc commutation failure condition, there are about 5 million system reactive power will be absorbed by the inverter side. If multiple dc commutation failure at the same time, a large number of reactive power will be absorbed from the system, and even cause damage to voltage stability.

\subsection{Short circuit capacity is insufficient in the weak-sending system}

With the development of the large energy base extended to outlying regions, especially the mass concentration and development of wind power and photovoltaic (pv), dc transmission sending power grid is weak and short circuit capacity shortage problem is prominent. On the one hand, the promotion of DC transmission capacity is depended heavily on the end of the thermal power, and the flexibility of the operation mode of the power grid and the consumption of the clean energy is impact directly. on the other hand, the transmitter system transient voltage will be rised due to the DC commutation failure, and the large-scale wind energy will be tripped under serious condition.

\subsection{Voltage stability problem on the load center}

Beijing, Tianjin and other regions of thermal power units will gradually shut down, part of the load from the gas supply unit. At the same time, with the further growth of the load, the proportion of external power will continue to increase. Due to the insufficient contradiction in voltage support, especially in the case of gas pipeline failure, will lead to gas units were forced outage, easy to lead to voltage stability, a serious threat to the power supply security in the capital area.

In summary, with the rapid development of UHVDC, large-scale development of clean energy, a large proportion of the concentration of power areas, the company's power grid characteristics of a large change in some areas of dynamic reactive power reserve decline, the problem of insufficient voltage support is prominent. Voltage stability problem has become one of the main problems of large and small power grid security and stability. It's means that the large-scale active DC transmission must match large-scale dynamic reactive power, that is, "large DC transmiss, strong reactive support".

In order to solve this problem, a large number of analytical studies have been carried out in the aspects of DC system voltage stabilization mechanism, AC / DC short circuit ratio, multi-feed transient voltage stabilization, dynamic reactive power support capability, generalized voltage safety warning and control system. The final scheme of the State Grid Corporation is decided to build a large-scale synchronous condenser to solve the voltage stability of the AC-DC hybrid system.

\section{Basic situation of the large synchronous condenser}

Synchronous condenser (also known as synchronous compensation machine) is a reactive power compensation device, is running in the motor state, to the power system to provide or absorb reactive power synchronous motor, without mechanical load without the prime mover. The grid structure and the secondary system of the large synchronous condenser is shown in figure 1.

Due to the high requirement of response speed and capacity in the UHV DC power transmission, the structure, dynamic characteristics, excitation control and relay protection and other aspects of the large synchronous condenser are different from generators and traditional synchronous condenser.

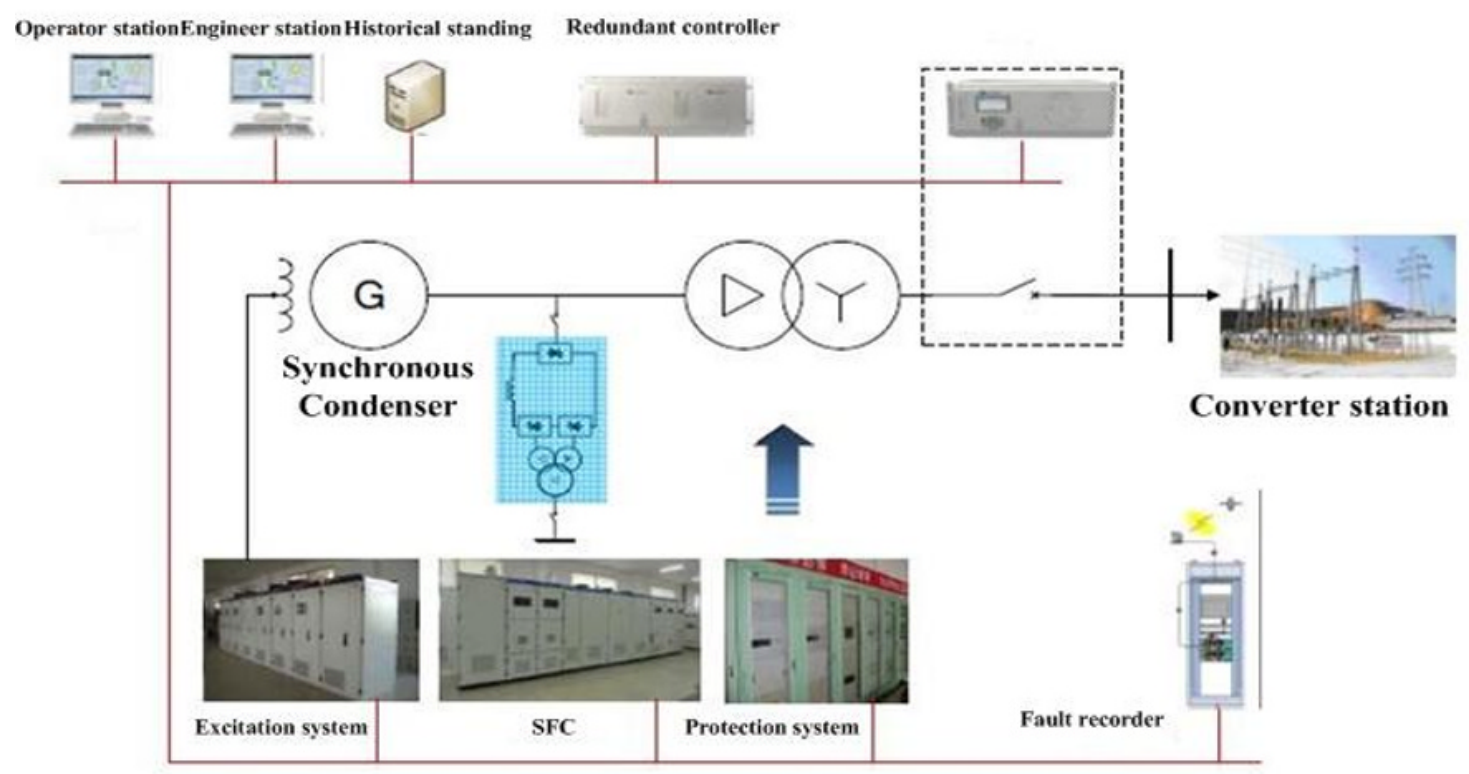

Fig.1. The grid structure and the secondary system of the large synchronous condenser. 


\section{Start mode of the static frequency conversion (SFC)}

Start excitation with the SFC, the unit is draged to 1.05 times the rated speed. The SFC exit is operated and the rated terminal voltage is established by the main excitation system. Then, the unit under idling process condition, the same period at the same time point of the grid. Starting mode of the SFC can be seen in fig.2. The process is as follows:

Step (1): under the cooperation of the excitation system, the SFC tractor unit rotor is accelerated by the turning state (except for the fast restart). During the startup process, the excitation system adopts its excitation circuit. Step (2): If the rotor of the large synchronous condenser is accelerated to $52.5 \mathrm{~Hz}$, the SFC system will exits. Then, the rotor began to degenerate due to loss of driving force. Step (3): In the process of unit inert speed down, excitation system will switch to the main excitation (the shunt) circuits and the start excitation system to exit. Step (4): After confirming that the SFC has been reliably disconnected from the stator, the excitation system enters the boosting process and establishes the rated terminal voltage. Step (5) :in the subsequent idle speed process, by the quasi-synchronization device to complete the same point of time to capture, and phase control unit into the grid.

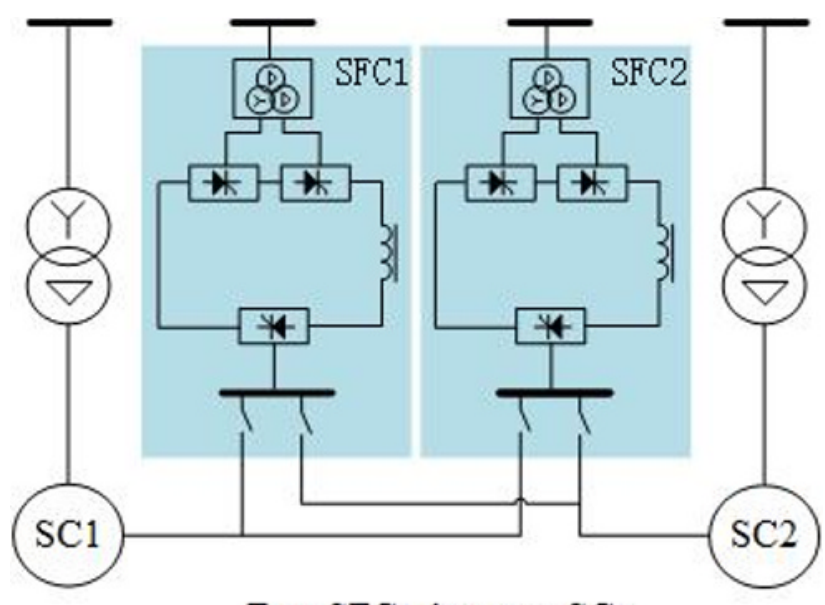

Two SFCs drag two SCs

Fig.2. Starting mode of the SFC.

\section{Excitation control system}

There are differences between the large-capacity tuner excitation system and generator excitation model, especially the extreme operating capacity of the large synchronous condenser under the low excitation and over-excitation conditions are different with the synchronous generator. Therefore, it is necessary to establish the special control model such as low-excitation and over-excitation of the large-capacity tuner excitation system. This paper studies the operation control strategy of the large capacity modulator under abnormal operating conditions and its exit mechanism under the extreme working condition, so as to give full play to the support of the large-scale modulator in the special condition of the grid voltage stability and dynamic reactive power.

\subsection{Performance requirements of excitation system}

Under power grid fault conditions, 3.5 times excitation voltage need to be provided when the terminal voltage is 0.8p.u.At the same time, the top value of the current needs to meet not less than 2.5 times, the time is not less than $15 \mathrm{sec}$ requirements, and its excitation voltage response time needs less than 0.1 sec.

\subsection{Control mode}

Under steady state condition, the steady-state operation is control by the high-voltage bus voltage and reactive power, and the transient operation is control by the voltage closed loop for fast excitation or reduction. Main ring of excitation control system is shown in fig.3.

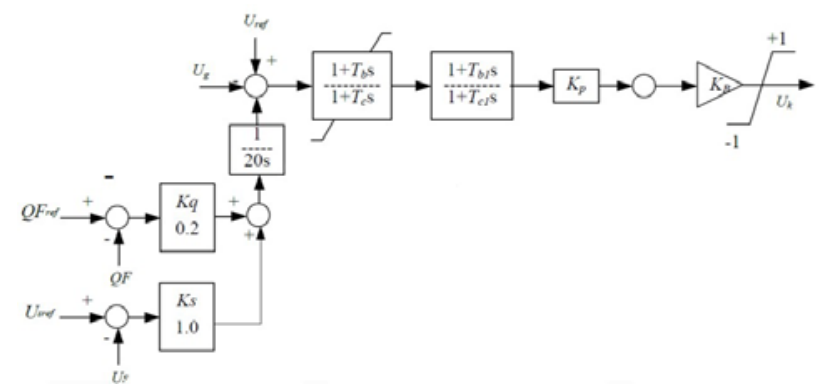

Fig.3. Main ring of excitation control system.

In addition, the fast reactive power control links is increased. The converter filter is cooperate with the synchronous condenser during the switching process. After received for the instruction, the Qref $q$ is the the aim to regulate in the synchronous condenser to achieve rapid reactive power output. The fast reactive power control links of excitation system are shown in fig. 4.

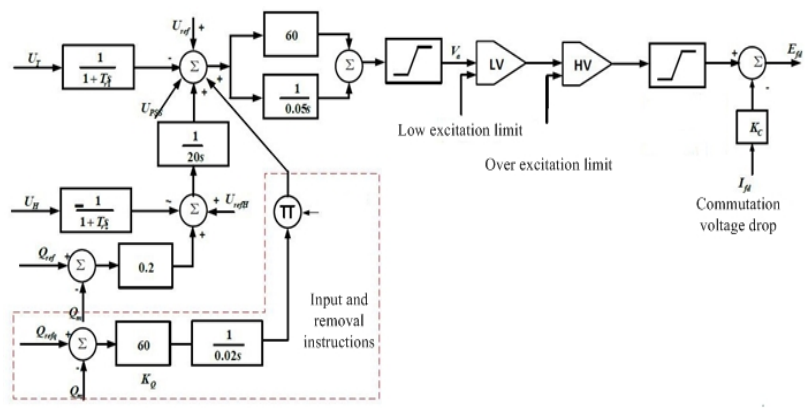

Fig.4. Fast reactive power control links of excitation system.

In addition, for the sending and receiving end grid, the characteristics of the high and low voltage operation under serious fault conditions, the influence of the structure and parameters of the excitation system on the rapid response performance of the large-scale synchronous condenser in different operating conditions 
needs to be considered. At the same time, for the many control strategies in the DC system rectification side and inverter side, the excitation control strategy of the synchronous condenser needs to meet the demand of rapid reactive power in the sending and receiving end power grid. The contrast of reactive power in synchronous condenser with or without cutting strategy can be seen in fig. 5 .

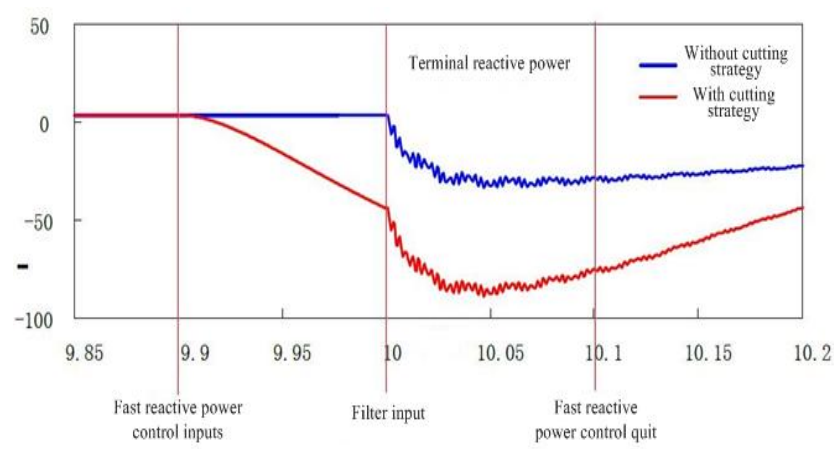

Fig.5. Contrast of reactive power in synchronous condenser with or without cutting strategy.

\section{Configuration and principle of relay protection}

The protective function of the large synchronous condenser are basically the same with the conventional generators. However, due to the special start-up and operation mode of the large synchronous condenser. The start torque of the SFC is provided by the synchronous condenser. The synchronous condenser is drag by stationary state to the rated speed condition. The corresponding unit electrical frequency varies rapidly from zero $\mathrm{Hz}$ to the rated frequency. After the synchronous condenser is connected to the grid, there is phase and late mode of operation, and the reactive power will be absorbed or send. Therefore, the principle and function configuration of the conventional protection group protection cannot be fully applied to the phase adjustment unit, is still need further study and analysis. Especially, in the protection in start process, loss of magnetic protection principle, as well as the protection in power grid and other aspects need further study.

\subsection{Protection on SFC start-up process}

Due to the large synchronous condenser is started with SFC, the low frequency characteristics of the electricity are present in protection device during start-up process. During the start-up process, the configuration and implementation of the protection scheme on the large synchronous condensers need to take into account the effects of low frequency characteristics. Some special functions can be configured to low frequency condition, such as, the low-frequency over-current protection, the differential protection and the stator ground fault protection in start-up process. Therefore, the independent of the frequency of the signal is necessary to be adopt in protection algorithms. In addition, the other protection of the large synchronous condenser may be mis-operation in the start-up process. Therefore, some measures can be used to prevent the mis-operation of the protection of the large synchronous condenser on start-up process.

\subsection{Loss of magnetic protection}

Conventional synchronous generator loss of magnetic protection of the stator impedance criterion is the use of static impedance impedance circle or asynchronous garden. However, the synchronous condenser has the ability to run without excitation. As a result, there will be no static stability and out-of-step problems when the synchronous condenser is out of force. In fact, the nonexcitation operation of the synchronous condenser will absorb a lot of reactive power from the system. In order to avoid a large number of reactive power converter caused the system power grid decline or even voltage instability problems, the configuration of the loss of magnetic protection can be constituted by the reactive components and the low-voltage components. In addition, in order to prevent the oscillation, short circuit or voltage circuit breakage and other abnormal circumstances of the protection of the situation, the corresponding latching components can be configured for the synchronous condenser.

\subsection{Protection of the grid with large synchronous condenser}

Due to the large-capacity two-way dynamic reactive power of the synchronous condenser, the original grid voltage distribution characteristics of the power system will be changed with the synchronous condenser. In particular, under the bidirectional dynamic reactive power condition, the inductive current or capacitive current will be provided by the synchronous condenser. It's means that the current distribution characteristics of the grid are changed. It could have new effects on AC current protection, distance protection and voltage protection. At the same time, the operation of the HVDC requires a lot of reactive power, the commutation voltage of the DC system can be maintained due to the Reactive power from the synchronous condenser. In addition, the probability of the inverter commutation failure is reduced when the bus voltage of the AC system is greatly reduced. Therefore, the impact of UHV DC protection with the large synchronous condenser is also an important concern.

In addition, compared with traditional synchronous generator, the standardization of relay protection design, configuration and setting technology of the large synchronous condenser are also changed. The standards for in a timely manner to carry out the work.

\section{Conclusion}

Due to the high requirement of response speed and capacity in the UHV DC power transmission system, the structure, dynamic characteristics, excitation control and relay protection and other aspects of the large synchronous condenser are different from generators and traditional synchronous condenser. Based on this, the 
dynamic reactive power demand of UHV DC power system is analyzed in this paper. Then, the main situation of large synchronous condenser is considered. In addition, the key points of the excitation control system and protection system are also discussed. Finally, the simulation model of the new-generation synchronous condensers access to UHV DC power grids are built based on the PSCAD software. The digital simulation results validate the transient reactive power support ability of the large synchronous condenser to UHVDC power transmission system. There are important theoretical and practical significance for the safe and reliable operation of the UHV DC/AC power system.

\section{Acknowledgment}

This work was supported by the State Grid Headquarters Science and Technology Project: Research on Fault Analysis, Relay Protection Technology and Operation Control Technology of the large synchronous condenser.

\section{References}

1. LIU Zhenya, ZHANG Qiping, WANG Yating, DONG Cun, ZHOU Qinyong. Research on Reactive Compensation Strategies for Improving Stability Level of Sending-end of $750 \mathrm{kV}$ Grid in Northwest China. Proceedings of the CSEE, 35 (2015)

2. Xu Zheng. Dynamic Behavior Analysis of AC / DC Power System. (Mechanical Industry Press, Beijing, 2005)

3. Zhang Jianshe, Zhang Yao, Zhang Zhichao, et al. Influence of dc system control modes on voltage andpower recovery after large disturbance in hybrid ac/dcsystems. Power System Technology, 29 (2005)
4. Yang Weidong, XuZheng, Han Zhenxiang. Coordinatedrecovery strategy for multi-infeed hvdcsystems. Electric Power Automation Equipment, 22 (2002)

5. Yang Weidong, XueYusheng, Jing Yong, et al. Acoordinated recovery strategy for 3 hvdc systems involvedin the south china power grid. Automation of ElectricPower Systems 27 (2003)

6. Wu Ping, Lin Weifang, Sun Huadong, et al. Research and electromechanical transient simulation on mechanism of commutation failure in multi-infeed hvdc power transmission system. Power System Technology, 36 (2012)

7. Ren Jing, Li Xingyuan, JinXiaoming, et al. Simulationstudy on commutation failure caused by switching acfilters of inverter stations in multi-infeed hvdcsystems. Power System Technology, 32 (2008)

8. JinXiaoming, Zhou Baorong, Guan Lin, eta. HVDCinteraction-strength index for the multi-infeed-hvdc power system. Automation of Electric Power Systems, 33 (2009)

9. Li Weifang, Tang Yong, Bo Guangquan. Definition and application of short circuit ratio for multi-infeed ac/dcpower systems. Proceedings of the CSEE, 28 (2008)

10. Sun Jingqiang, GuoXiaojiang, Zhang Jian, et al. Dynamic characteristics of receiving-end of multiinfeed hvdcpower transmission system. Power System Technology, 33(2009)

11. Zhou Dongxia,YuRui, etc. SFC start and relay protection of 300 Mvar large synchronous condenser. Power System Protection and Control, 20 (2016) 